\title{
COVID-19: an In Silico Analysis on Potential Therapeutic Uses of Trikadu as Immune System Boosters
}

\author{
Rukaiya Hasani Khasamwala $^{1}$ · S. Ranjani ${ }^{1}$ - S. Sai Nivetha ${ }^{1}$ - S. Hemalatha ${ }^{1}$
}

Accepted: 30 November 2021 /

Published online: 6 January 2022

(c) The Author(s), under exclusive licence to Springer Science+Business Media, LLC, part of Springer Nature 2021

\begin{abstract}
Corona virus pandemic outbreak also known as COVID-19 has created an imbalance in this world. Scientists have adopted the use of natural or alternative medicines which are consumed mostly as dietary supplements to boost the immune system as herbal remedies. India is famous for traditional medicinal formulations which includes 'Trikadu' - a combination of three acrids, namely Zingiber officinale, Piper nigrum and Piper longum which have antioxidant properties that boost our immune system hence acting as a strong preventive measure. In this study, AutoDock 4.0 was used to study interaction between the phytocompounds of Trikadu with RNA-dependent polymerase protein and enveloped protein of the SARS-CoV-2 virus. Analysis of the results showed that coumarin, coumaperine and bisdemethoxycurcumin showed strong bonding interactions with both the proteins. We can conclude that Trikadu has the potential molecules; hence, it can be incorporated in the diet to boost the immune system as a preventive measure against the virus.
\end{abstract}

Keywords SARS-coV-2 - Immune response · Trikadu · Ginger · Pepper · Long pepper · Protein-ligand interactions

\section{Introduction}

The coronavirus (COVID-19) is an infectious disease-causing respiratory ailment affecting the lungs caused by a novel strain of virus called severe acute respiratory syndrome coronavirus 2 (SARS-CoV-2). Corona viruses are a group of viruses found in many species including cattle, camel and bats which are responsible for the outbreak of respiratory illness [1]. The virus has a main presenting symptom which is pneumonia and is suspected of having originated from a local seafood market whose source is an unknown animal most likely assumed to be bat for being responsible for the emergence and spread of the novel strain of the coronavirus [2].

Rukaiya Hasani Khasamwala and S. Ranjani contributed equally to this work

S. Hemalatha

hemalatha.sls@bsauniv.ac.in

1 School of Life Sciences, B. S. Abdur Rahman Crescent Institute of Science and Technology, Vandalur, Chennai, India 600048 
The World Health Organization (WHO) along with the scientists have adopted using alternative traditional medicine which has benefits and working with institutions in selecting the traditional medicines which are showing high level of efficacy in clinical trials and using it as a treatment against COVID-19 [3-5].

The immune system provides a strong protection against foreign pathogens and by boosting it, we can strengthen the line of defence mechanism. We can avoid the immune weakening food and instead adding immune boosting food to our diet helps in providing strong defence mechanism to our body. This will avoid our body to be in a state of inflammation which further weakens our immune system. Avoiding processed, trans fat and excessive carbohydrate-rich foods and by increasing daily consumption of plant-based food will increase antioxidants in our body and combat oxidative stress which in turn boost mitochondrial and cellular functions because spices are highly antioxidant and immune protective [6].

We have chosen 'Trikadu', for this study which is a mixture of three acrids ginger (Zingiber official Roscoe), pepper (Piper nigrum) and long pepper (Piper longum). Trikadu have antioxidant and anti-inflammatory properties. The active phytocompounds of Trikadu help in boosting the immune system by minimizing the inflammation. Hence, in Trikadu, the combination of these effective phytocompounds can be used as potential agents in combating the virus and aiding in faster recovery. Herbs are still found in $40 \%$ of the prescribed medicines, and the interest in using herbal remedies is preferred over chemical drugs due to less side effects in humans as it is in accordance with nature in its raw composition [7].

Ginger (Zingiber official Roscoe) belongs to the family Zingiberaceae is famous for its anti-inflammatory properties and is widely used as a medicinal product that is herbal in nature and which also shares similarities with anti-inflammatory drugs that are non-steroidal in nature. Extracts of ginger consist of a complex multicomponent mixture of biologically active components including compounds which are structurally similar like gingerols, paradols and shogaols which account majorly for the anti-inflammatory properties [8].

Black pepper (Piper nigrum) is a native plant to the tropical regions of India and is also known as the 'King of Spices'. It is used in Ayurveda, Unani and Siddha for thousands of years. According to Ayurveda, the pungency and the heating properties of the pepper help in metabolizing food as it helps in digesting it. The heat acts as a stimulant which clears congestion in the respiratory system [9].

Long pepper (Piper longum) is used in the traditional system of Ayurvedic medicine. It is most commonly used to treat diarrhoea, cholera, stomachache and respiratory infections. Long pepper is closely related to $P$. nigrum and comparatively has a hot flavour. The odour is peculiar and produces a very strong pungent taste which leaves the tongue numb. The piperine content is around 3-5\% in P. longum [10].

Gallic acid, kaempferol, demethoxycurcumin, coumarin, coumaperine, bisdemethoxycurcumin, gingerol, (10)-gingerol, (8)-gingerol, shogaol and paradol are the major phenolic compounds present in the Trikadu. Coumarin (2H-1-Benzopyran-2-one) is a major phenolic substance present in plants. They are commonly present in essential oils, green tea and chicory. Coumarin is a constituent of pepper plant and is used in anti-inflammatory activity, anticoagulant, antibacterial, antifungal, antiviral and anticancerous. They have wide pharmacological uses and can be used as a novel therapeutic target for ailments [11]. Demethoxycurcumin and bisdemethoxycurcumin are the ingredients of long pepper. They have a potential for curing many diseases affecting the liver, Alzheimer's disease and Parkinson's. They possess anti-inflammatory, antiviral, antibacterial and antioxidant properties. Demethoxycurcumin and bisdemethoxycurcumin give the yellow colour to the plant [12]. 
The spike protein mediates the fusion of the viral and host membrane which contains a receptor binding domain (RBD) that attaches itself to the cell during the entry of the virus [13]. The receptor of SARS-CoV-2 is an angiotensin converting enzyme (ACE-2). The spike proteins involved in the cycle of virus include 6LXT which is an enveloped protein that plays a role in budding, fusion of envelope and pathogenesis that are involved in the life cycle of virus [14]. 6M71 protein is a SARS-CoV-2 and is an RNA-dependent RNA polymerase in complex with cofactors. The RNA-dependent RNA polymerase is the central component of the viral replication and transcription machinery; it also appeared to be the primary target of the antiviral drug remdesivir $[15,16]$.

Hence, these two proteins were selected to perform in silico analysis using molecular docking with the 11 bio-active phenolic components (ligands) of Trikadu, a mixture of three spices, and to study their potential therapeutic effectiveness and the scope of using it as an immune booster in combating the virus and an effective alternative remedy with no side effects in case of immuno-suppressed individuals too.

\section{Materials and Methods}

The 3D structures of ligands including gallic acid, kaempferol, demethoxycurcumin, coumarin, coumaperine, bisdemethoxycurcumin, gingerol, (10)-gingerol, (8)-gingerol, shogaol, paradol were obtained from PubChem in SDF format and converted to PDB format by Open Babel disciple to facilitate molecular docking analysis. The two proteins 6LXT which is a structure of post fusion core of 2019-nCoV S2 subunit whose resolution is $2.90 \AA$ by X-ray diffraction and 6M71 which is a SARS-Cov-2 RNA-dependent RNA polymerase in complex with cofactors whose resolution is $2.90 \AA$ by electron microscopy are docked with the phenolic phytocompounds of ginger, pepper and long pepper using the software Marvin view, AutoDock 4.0, BIOVIA Discovery studio and PyMol for simulation and visualization [17]. The best conformational pose was decided based on the RMSD table. The best conformations were observed using AutoDock which shows number of hydrogen bonds. Interaction poses with more number of hydrogen bonds were considered to be more stable interaction [18].

\section{Molecular Docking}

Protein and ligand preparation was done using AutoDock after downloading the structures from Protein Data Bank (PDB) and PubChem. Free-energy scoring function based on linear regression analysis and AMBER force field give an RMSD table which gives summary of the binding energies based on different interactions. This provides good quality of conformations of ligands and a strong correlation between the constant of inhibition and the experimental ones. The water molecules are removed from the proteins, so it does not interfere during the binding using AutoDock. Polar hydrogen was added using AutoDock while preparing the protein. The grid was prepared using Blind Docking, and AutoGrid was run, which gives the electrostatic potential maps and desolvation maps which are used by AutoDock to guide the docking process using the active site as the site of binding. AutoDock was then run which gives the RMSD table that consists of rank, sub-rank, run, binding energy, cluster RMSD, reference RMSD and Grep pattern for each run. The runs with the highest ranking and minimum binding energies were selected. The interaction for the run with best binding energy was visualized using BIOVIA Discovery studio. This helps us 
to identify the number of hydrogen bonds formed between the protein and ligand and gives information about the interacting amino acids.

\section{Results and Discussion}

The protein structures were obtained from PDB and docked with 11 compounds of ginger, pepper and long pepper of the Trikadu mixture. The AutoDock gave the possible conformations of the ligand as well as the RMSD table which gave the binding affinities of the protein and the ligand. The conformations for each ligand were further analyzed by analysing the number of hydrogen bonds formed to check the interactions with the target proteins and find out the amino acids involved in the interactions. PubChem ID, binding energy, root mean square division values and residues of target ligand molecule which interact with the proteins are given in the tables (Tables 1 and 2) below. These interactions were observed using BIOVIA Discovery studio to visualize the hydrogen bonds and the amino acids involved.

\section{Molecular Docking Studies}

Molecular docking studies of both the proteins (6LXT and 6M71) were docked with 11 phenolic phytocompounds of the Trikadu mixture which acts as an immune booster that enables the first line of defence to boost the immune system (Supplementary Fig. 1, Supplementary Fig. 2).

Based on the results of molecular docking studies, 6LXT post fusion core of 2019nCoV S2 subunit showed the best binding energy with coumarin (Fig. 1) and 6M71SARSCov-2 RNA-dependent RNA polymerase showed the best binding energy with coumaperine (Fig. 2).

From the comparative analysis (Fig. 3), we can observe that the binding energy of the "coumarin" (323) compound was the lowest with the 6LXT (enveloped protein) that is $-6.44 \mathrm{kcal} / \mathrm{mol}$ and the highest binding energy was of the interaction between (10)-gingerol $(168,115-6 \mathrm{LXT})$ and 6LXT (enveloped protein) which was $-3.10 \mathrm{kcal} / \mathrm{mol}$. Hence, coumarin has the most stable interaction followed by coumaperine $(10,131,321)$, demethoxycurcumin $(5,469,424)$ and bisdemethoxycurcumin $(5,315,472)$ showing that they were more effective in blocking the active site of the protein by interacting with them, indirectly leading to boosting the immune system and making it immune towards the virus.

These spices have been placed in the category of functional foods which are known for their health benefits in boosting the immune system [19, 20]. These phytocompounds have antioxidant properties needed and also prevent food rancidity hence making the consumption safe [21]. Previous research also concludes that ginger is a good source as an antioxidant and can be used as a supplement to boost the immune system [19].

From the comparative analysis done in Fig. 4, we can see that the binding energy of the coumaperine $(10,131,321)$ and bisdemethoxycurcumin $(5,315,472)$ was the lowest being $-7.50 \mathrm{kcal} / \mathrm{mol}$ and $-7.48 \mathrm{kcal} / \mathrm{mol}$, respectively. This shows that the two ligands were highly stable and require the least binding energy to interact with the 6M71 (RNAdependent RNA polymerase protein). Following them are demethoxycurcumin $(5,469,424)$ and coumarin (323).wW 


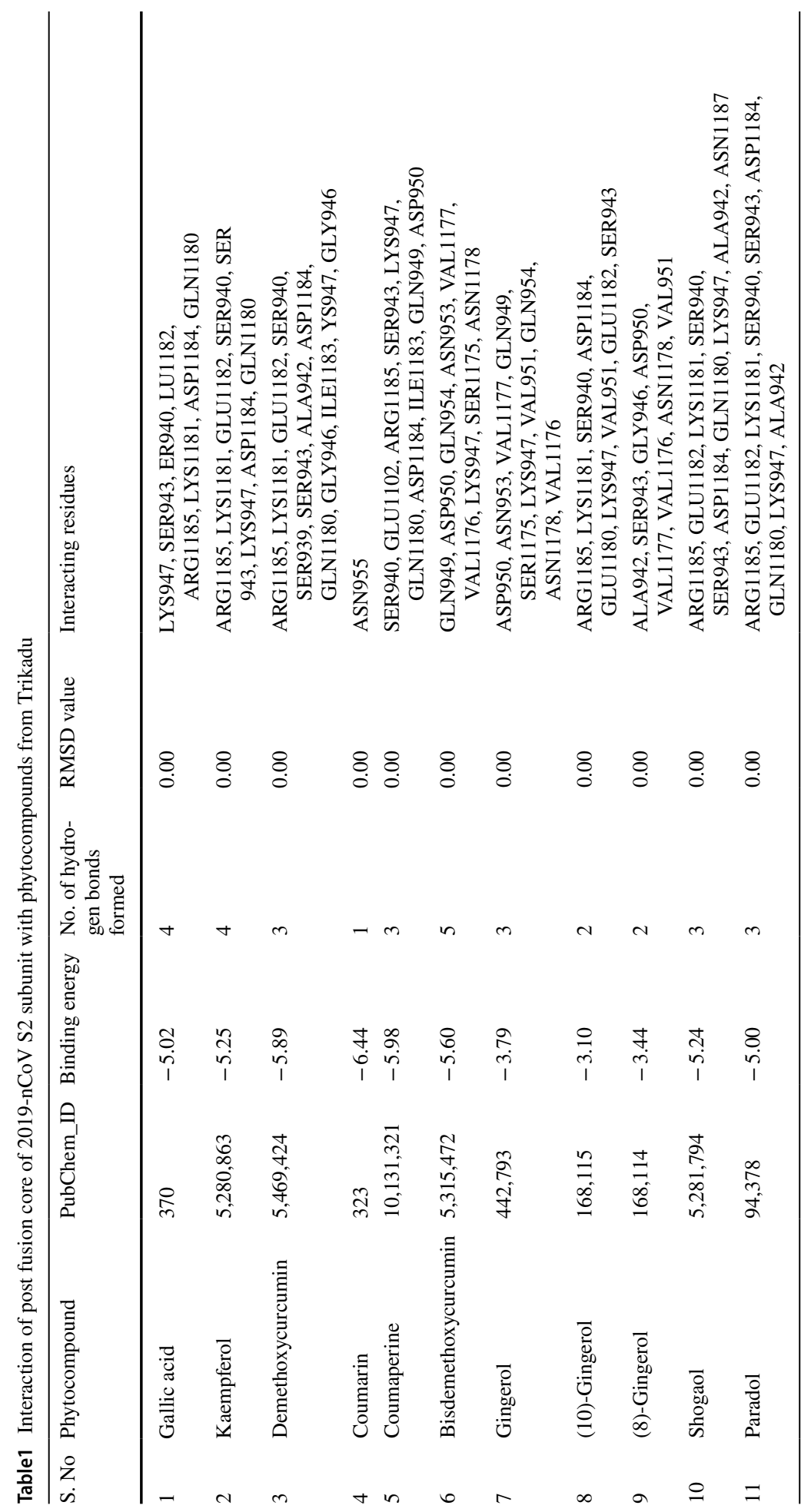




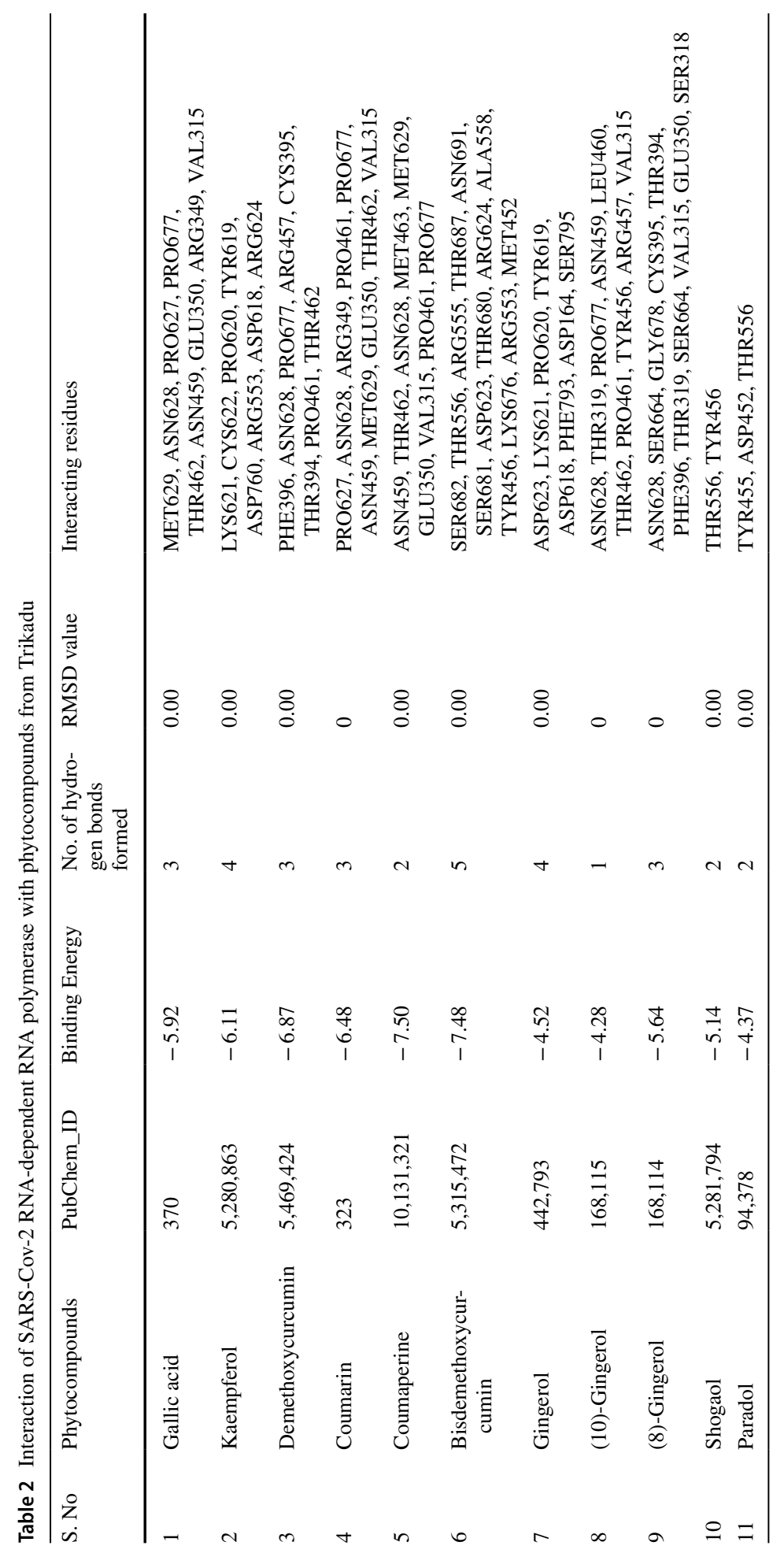


Fig. 1 Interaction of post fusion core of 2019-nCoV S2 subunit with coumarin

Fig. 2 Interaction of RNAdependent RNA polymerase protein with coumaperine
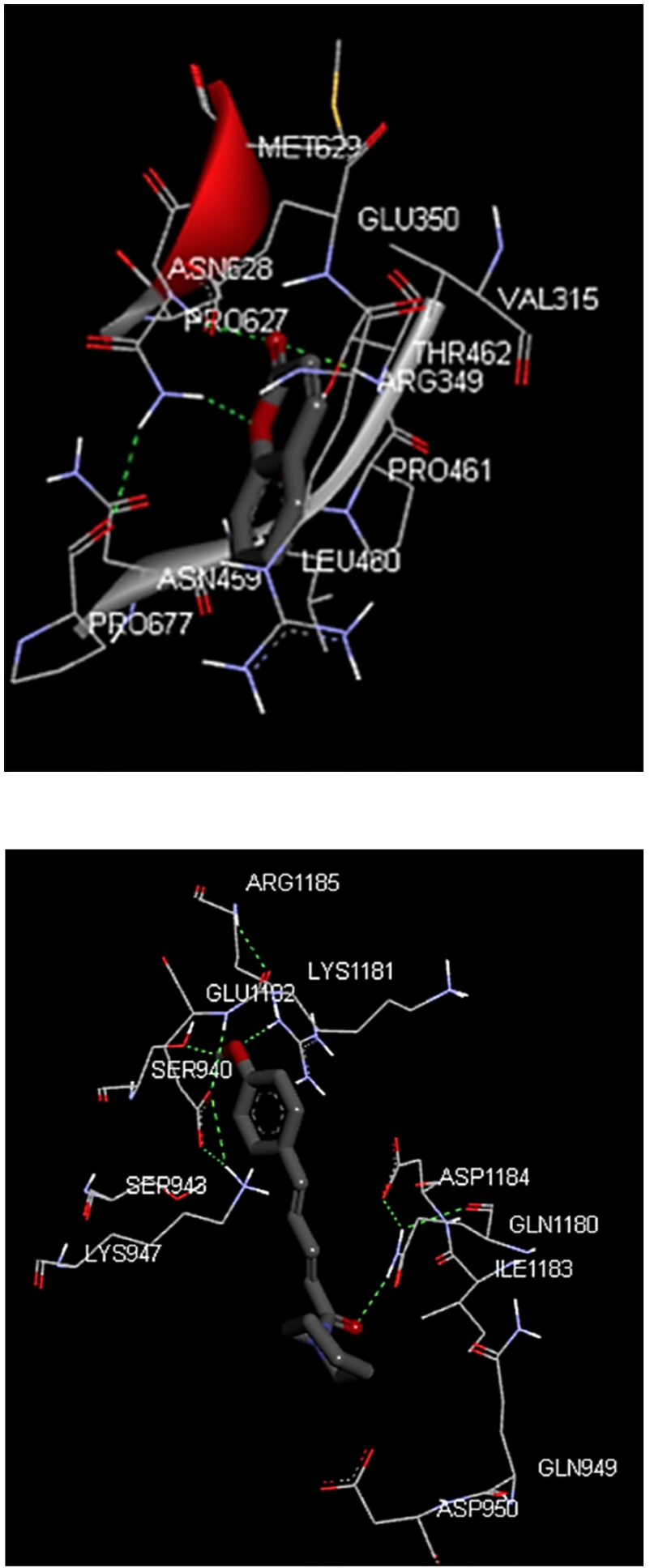


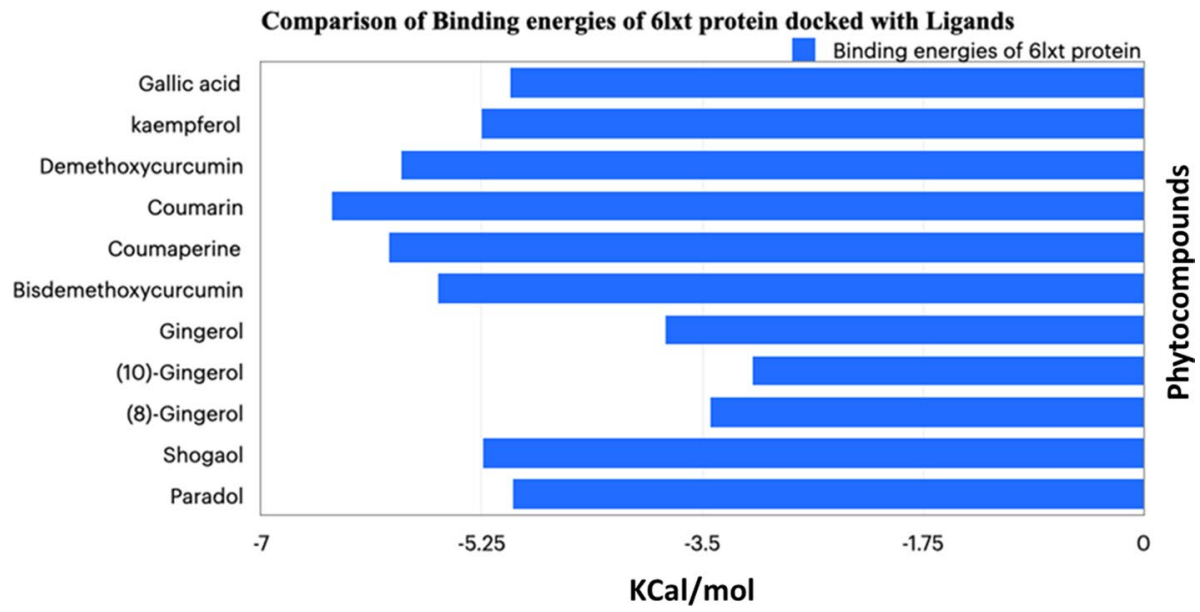

Fig. 3 Binding energies of post fusion core of 2019-nCoV S2 subunit protein with the compounds

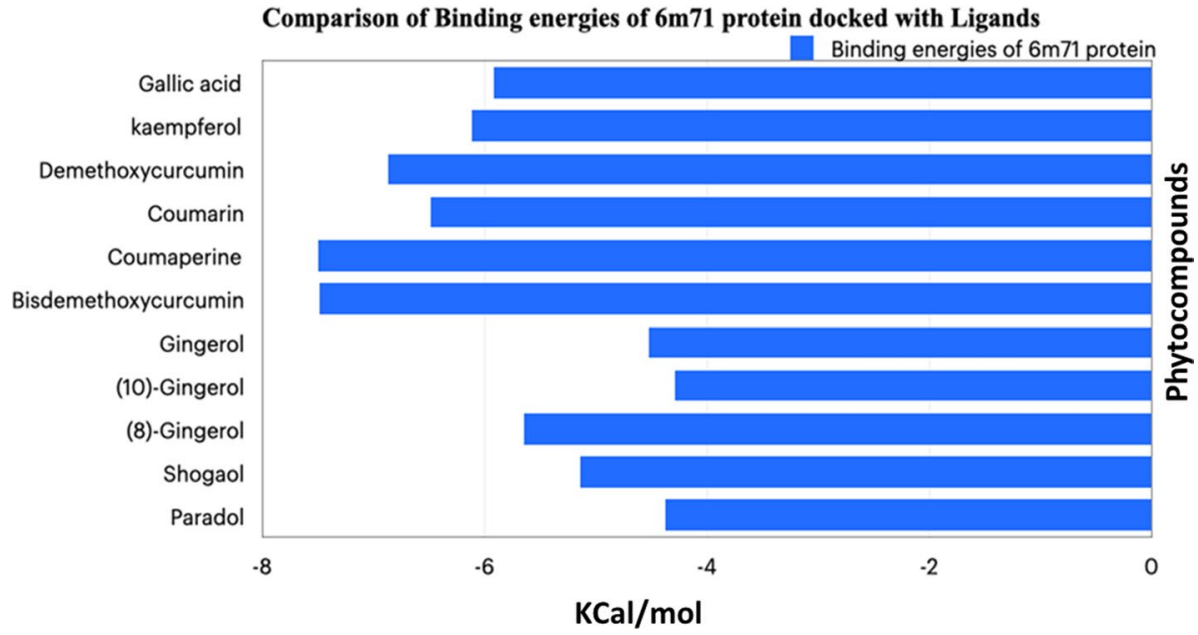

Fig. 4 Binding energies of RNA-dependent RNA polymerase protein with the compounds

From the analysis of results, we can see that the compounds coumarin, coumaperine and bisdemethoxycurcumin show good binding activity and are an active component of pepper and ginger, respectively. The highest binding energy is again of (10)-gingerol which means it has the least number of hydrogen bonds formed with both the proteins of 6LXT and 6M71 but it is unstable. The best binding molecule, coumarin, showed interaction with amino acid residues such as ASN955 with 6LXT protein and PRO627, ASN628, ARG349, PRO461, PRO677, ASN459, MET629, GLU350, THR462 and VAL315 with 6M71 protein which is shown in Tables 1 and 2.

These docking studies give the information of binding potential of phenolic compounds with both the target protein. All the compounds were more effective in blocking 
the protein and can be effective as a preventive measure against COVID-19 and can be consumed on regular levels to boost the immune system. The phytocompounds present in pepper is an excellent source for harnessing therapeutic effects using bioavailability of the drug metabolism. The oxidative damage is protected by quenching of free radicals which in turn increases the antioxidants and prevents oxidative stress. It also activates the delay of procarcinogens growth by using drug metabolizing systems hence being a potential antitumor agent [22]. The phytocompounds of long pepper (Piper longum) are used extensively in Ayurveda, Unani and Siddha for disease related to respiratory tracts [23].

This study is performed to demonstrate the efficacy of phytocompounds of Trikadu. Hence, consumption of Trikadu can reduce the severity of the symptoms of COVID-19 virus. Trikadu functions as a natural immune booster using natural spices of ginger (Zingiber officinale), pepper (Piper nigrum) and long pepper (Piper longum) in equal ratio. These spices are responsible and mainly involved in boosting the immune system by possessing strong anti-inflammatory and antioxidant properties which increases the immune response and acts as a second line of defence by keeping the body enriched with the required antioxidants. COVID-19 is linked to the diet, and maintaining a healthy diet during a pandemic increases the survival of the host and keeps the body healthy and not in an inflamed state. The future scope of the study includes formulating an antioxidant drink which consists of the spices ginger, pepper and long pepper and the combination of all the three spices. This formulated powerful drink can be used in adequate dosage and consumed on a regular basis to boost the immune system and keep the body enriched with antioxidants and in an anti-inflammatory which will promote faster healing and increase the production of antibodies in blood. The future work also includes using different spices and performing an in silico analysis to find the scope of the natural spices whose bioavailability is high and to provide second line of defence in avoiding many health issues and increasing the lifespan simultaneously. Hence, this in silico analysis showed the scope of therapeutic effectiveness of Trikadu combination of three acrids ginger, pepper and long pepper in battling COVID-19 and controlling the pandemic.

\section{Conclusion}

This docking study using the phytocompounds of Trikadu shows that they are more effective in blocking the protein of COVID19. Thus, Trikadu can be an effective preventive measure against COVID-19 and can be consumed on regular levels to boost the immune system by possessing strong anti-inflammatory and antioxidant and also acts as a second line of defence by keeping the body enriched with the required antioxidants. COVID-19 is linked to the diet, and maintaining a healthy diet during pandemic keeps the body healthy.

Supplementary Information The online version contains supplementary material available at https://doi. org/10.1007/s12010-021-03793-5.

Acknowledgements All authors are thankful to B.S. Abdur Rahman Institute of Science \& Technology, Chennai for providing research facilities in school of life sciences. The authors also gratefully acknowledge the Ministry of Science and Technology, Department of Science and Technology (KIRAN Division) (GoI), New Delhi. (Ref No. DST/WOS-B/2018/1583-HFN (G)) and DST/SATYAM/COVID-19/2020/213 (G).

Author Contribution SH conceived and designed research. SR, SRHK, and SNS conducted computational experiments. All authors wrote the manuscript. All authors read and approved the manuscript. 
Data Availability Data will be available on request.

Code Availability Not applicable.

\section{Declarations}

Ethics Approval Not applicable.

Consent to Participate Not applicable.

Consent for Publication All authors read and approved the manuscript for publication.

Competing Interests The authors declare no competing interests.

\section{References}

1. Pal, M., Berhanu, G., Desalegn, C., \& Kandi, V. (2020). Severe acute respiratory syndrome coronavirus-2 (SARS-CoV-2): An update. Cureus, 12(3), e7423.

2. Singhal, T. (2020). A Review of coronavirus disease-2019 (COVID-19). Indian Journal of Pediatrics, 87(4), 281-286.

3. Ang, L., Song, E., Lee, H. W., \& Lee, M. S. (2020). Herbal medicine for the treatment of coronavirus disease 2019 (COVID-19): A systematic review and meta-analysis of randomized controlled trials. Journal of Clinical Medicine, 9(5), 1583.

4. Lin, L. T., Hsu, W. C., \& Lin, C. C. (2014). Antiviral natural products and herbal medicines. Journal of Traditional and Complementary Medicine, 4(1), 24-35.

5. Kiran, G., Karthik, L., Shree Devi, M. S., Sathiyarajeswaran, P., Kanakavalli, K., Kumar, K. M., \& Ramesh, K. D. (2020). In Silico computational screening of Kabasura Kudineer - Official Siddha Formulation and JACOM against SARS-CoV-2 spike protein. Journal of Ayurveda and Integrative Medicine, S0975-9476(20), 30024-30033.

6. Yashin, A., Yashin, Y., Xia, X., \& Nemzer, B. (2017). Antioxidant activity of spices and their impact on human health: A review. Antioxidants (Basel), 6(3), 70.

7. Ekor, M. (2014). The growing use of herbal medicines: Issues relating to adverse reactions and challenges in monitoring safety. Frontiers in Pharmacology, 4, 177.

8. Grzanna, R., Lindmark, L., \& Frondoza, C. G. (2005). Ginger--An herbal medicinal product with broad anti-inflammatory actions. Journal of Medicinal Food, 8(2), 125-132.

9. Salehi, B., Zakaria, Z. A., Gyawali, R., et al. (2019). Piper species: A comprehensive review on their phytochemistry, biological activities and applications. Molecules, 24(7), 1364.

10. Kumar, S., Kamboj, J., Suman, \& Sharma, S. (2011). Overview for various aspects of the health benefits of Piper longum linn. fruit. Journal of Acupuncture and Meridian Studies, 4(2), 134-140.

11. Leyva-Jiménez, F. J., Lozano-Sánchez, J., Cádiz-Gurrea, M. L., Arráez-Román, D., \& Segura-Carretero, A. (2019). Functional Ingredients based on Nutritional Phenolics. A Case Study against Inflammation: Lippia Genus. Nutrients, 11(7), 1646.

12. Sandur, S. K., Pandey, M. K., Sung, B., et al. (2007). Curcumin, demethoxycurcumin, bisdemethoxycurcumin, tetrahydrocurcumin and turmerones differentially regulate anti-inflammatory and anti-proliferative responses through a ROS-independent mechanism. Carcinogenesis, 28(8), 1765-1773.

13. Belouzard, S., Millet, J. K., Licitra, B. N., \& Whittaker, G. R. (2012). Mechanisms of coronavirus cell entry mediated by the viral spike protein. Viruses, 4(6), 1011-1033.

14. Du, L., He, Y., Zhou, Y., Liu, S., Zheng, B. J., \& Jiang, S. (2009). The spike protein of SARS-CoV-A target for vaccine and therapeutic development. Nature Reviews Microbiology, 7(3), 226-236.

15. Gao, Y., Yan, L., Huang, Y., et al. (2020). Structure of the RNA-dependent RNA polymerase from COVID-19 virus. Science, 368(6492), 779-782.

16. Chaw, S., Tai, J., Chen, S., et al. (2020). The origin and underlying driving forces of the SARS-CoV-2 outbreak. Journal of Biomedical Science, 27, 73.

17. Elfiky, A. A. (2020). Ribavirin, Remdesivir, Sofosbuvir, Galidesivir, and Tenofovir against SARSCoV-2 RNA dependent RNA polymerase (RdRp): A molecular docking study. Life Sciences, 253, 117592.

18. Pantsar, T., \& Poso, A. (2018). Binding affinity via docking: Fact and fiction. Molecules, 23(8), 1899. 
19. Mao, Q. Q., Xu, X. Y., Cao, S. Y., et al. (2019). Bioactive compounds and bioactivities of ginger (Zingiber officinale Roscoe). Foods, 8(6), 185.

20. Serafini, M., \& Peluso, I. (2016). Functional foods for health: The interrelated antioxidant and antiinflammatory role of fruits, vegetables, herbs, spices and cocoa in humans. Current Pharmaceutical Design, 22(44), 6701-6715.

21. Lee, M. T., Lin, W. C., Yu, B., \& Lee, T. T. (2017). Antioxidant capacity of phytochemicals and their potential effects on oxidative status in animals - A review. Asian-Australasian Journal of Animal Sciences, 30(3), 299-308.

22. Takooree, H., Aumeeruddy, M. Z., Rengasamy, K. R. R., Venugopala, K. N., Jeewon, R., Zengin, G., \& Mahomoodally, M. F. (2019). A systematic review on black pepper (Piper nigrum L.): from folk uses to pharmacological applications. Critical Reviews in Food Science and Nutrition, 59(sup1), S210-S243.

23. Pandey, M. M., Rastogi, S., Rawat, A. K. (2013). Indian traditional ayurvedic system of medicine and nutritional supplementation. Evidence-Based Complementary and Alternative Medicine, 2013, 376327.

Publisher's Note Springer Nature remains neutral with regard to jurisdictional claims in published maps and institutional affiliations. 